\title{
Intraoral ultrasonography in the assessment of DOI in oral cavity squamous cell carcinoma: a comparison with magnetic resonance and histopathology
}

\author{
Marta Filauro ${ }^{1,2,3} \cdot$ Francesco Missale $^{1,4} \cdot$ Filippo Marchi $^{1,3} \cdot$ Andrea landelli $^{1} \cdot$ Andrea Luigi Camillo Carobbio $^{1,2}(\mathbb{D}$. \\ Francesco Mazzola ${ }^{1,2}$. Giampiero Parrinello ${ }^{1}$. Emanuele Barabino ${ }^{1,5} \cdot$ Giuseppe Cittadini $^{1,5} \cdot$ Davide Farina $^{6}$. \\ Cesare Piazza ${ }^{7}$. Giorgio Peretti ${ }^{1,2}$
}

Received: 8 September 2020 / Accepted: 5 October 2020 / Published online: 21 October 2020

(c) The Author(s) 2020

\begin{abstract}
Objective The first-line therapeutic approach for oral cavity squamous cell carcinoma (OCSCC) is complete surgical resection. Preoperative assessment of depth of invasion (cDOI) is crucial to plan the surgery. Magnetic resonance (MR) and intraoral ultrasonography (IOUS) have been shown to be useful tools for assessment of DOI. The present analysis investigates the accuracy of MR and IOUS in evaluating DOI in OCSCC compared to histological evaluation (pDOI).

Materials and methods Forty-nine previously untreated patients with cT1-T3 OCSCC were reviewed. Nine patients were staged with MR alone, 10 with IOUS alone, and 30 with both MR and IOUS.

Results Mean difference between $\mathrm{cDOI}_{\mathrm{MR}}$ and pDOI values of $0.2 \mathrm{~mm}(95 \% \mathrm{CI}-1.0-1.3 \mathrm{~mm})$ and between cDOI $\mathrm{IOUS}_{\text {and }}$ pDOI of $0.3 \mathrm{~mm}(95 \% \mathrm{CI}-1.0-1.6 \mathrm{~mm})$. Spearman $R$ between $\mathrm{cDOI}_{\mathrm{MR}}$ and $\mathrm{pDOI}$ was $R=0.83$ and between $\mathrm{cDOI}_{\mathrm{IOUS}}$ and pDOI was $R=0.76$. Both radiological techniques showed high performance for the correct identification, with the optimum cut-off of $5 \mathrm{~mm}$, of patients with a pDOI $\geq 4 \mathrm{~mm}$ and amenable to a neck dissection, with an AUC of 0.92 and 0.82 for MR and IOUS, respectively.

Conclusion Both examinations were valid approaches for preoperative determination of DOI in OCSCC, although with different cost-effectiveness profiles and indications.
\end{abstract}

Keywords Mouth $\cdot$ Neoplasm $\cdot$ Magnetic resonance imaging $\cdot$ Ultrasonography $\cdot$ Depth of invasion $\cdot$ Head and neck

\section{Introduction}

Electronic supplementary material The online version of this article (https://doi.org/10.1007/s00405-020-06421-w) contains supplementary material, which is available to authorized users.

Andrea Luigi Camillo Carobbio

a.carobbio@studenti.unibs.it

1 IRCCS Policlinico San Martino, Largo Rosanna Benzi, 10, 16132 Genoa, Italy

2 Interdisciplinary Department of Surgical and Integrated Diagnostic Sciences (DISC), University of Genoa, Genoa, Italy

3 Department of Experimental Medicine (DIMES), University of Genoa, Genoa, Italy
Head and neck cancer is the 6th most common malignancy worldwide, and oral cavity tumors account for nearly onethird of the tumors [1]. Squamous cell carcinoma (SCC) is the most frequent histotype: it usually arises in the mobile tongue, followed by the lip, floor of the mouth, and buccal

4 Department of Molecular and Translational Medicine, University of Brescia, Brescia, Italy

5 Department of Diagnostic Radiology, IRCCS Policlinico San Martino, Genoa, Italy

6 Department of Radiology, University of Brescia, Brescia, Italy

7 Department of Otorhinolaryngology, Maxillofacial, and Thyroid Surgery, Fondazione IRCCS, National Cancer Institute of Milan, University of Milan, Milan, Italy 
mucosa. There is a male preponderance and the vast majority of patients are heavy smokers and alcohol abusers.

Novel relevant changes in the $\mathrm{T}$ classification of oral cavity SCC (OCSCC) were brought about by the 8th Edition of the AJCC UICC TNM Staging System [2]. Neoplastic depth of invasion (DOI) was introduced as one of the main aspects to be considered during tumor staging. DOI is defined as "the deepest invasion of tumor in the tissue from the mucosal surface or from a theoretical reconstructed normal mucosal line" [3]. Thus, it profoundly differs from "tumor thickness" (TT), the latter being defined as the distance of the tumor surface from the deepest level of invasion [4]. Consequently, DOI may result in appreciably less TT in exophytic lesions and higher in ulcerated ones, while the two values may correspond in case of fundamentally flat tumors. It is crucial to precisely evaluate the tendency of the tumor to infiltrate healthy tissue since this characteristic reflects the presence of regional lymph node metastases. Thus, recognizing which radiological examination performs better in giving precise preoperative assessment of DOI is of utmost value. However, definitive DOI estimation can be obtained only from measures performed at final histopathological examination (per se subjected to inconsistent variations in terms of tissue shrinkage due to chemical fixation), even if the possibility to have an adequate preoperative evaluation of this parameter allows the surgeon to properly devise the surgical resection (e.g. choosing between transoral resection or compartmental surgery with flap reconstruction) as well as the need for elective neck dissection (strongly suggested for OCSCC with DOI $\geq 4 \mathrm{~mm}$ ) [5-7]. Moreover, preoperative radiologic measurement of DOI gives the surgeon essential information to tailor the resection within free margins, with particular reference to the deepest one, which represents a significant treatment-related predictor in terms of diseasefree survival and loco-regional control [8].

This study aims to identify the best radiological examination for patients with OCSCC in assessing tumor staging, with special reference to DOI, and, consequently, to tailor the best surgical treatment in terms of oncological radicality on $\mathrm{T}$ and $\mathrm{N}$ sites. In particular, a direct comparison was performed between magnetic resonance (MR), one of the gold standards in modern OCSCC preoperative imaging [9], and intraoral ultrasonography (IOUS), an emerging technique with a new profile of interest.

\section{Materials and methods}

We retrospectively collected data from 49 patients affected by cT1-T3 OCSCC treated at our institution between April 2016 and October 2019.

Inclusion criteria encompassed: (1) clinical evidence and biopsy confirmation of a previously-untreated OCSCC; (2) preoperative evaluation of the lesion by IOUS and/or MR; (3) complete excision of the lesion to obtain its final histopathological evaluation and tumor measurement. Tumor clinical DOI (cDOI) was measured on MR ( $\left.\mathrm{cDOI}_{\mathrm{MR}}\right)$ and

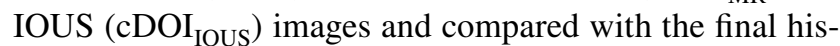
topathological measure (pDOI).

Preoperative radiological assessment of tumor size, together with clinical inspection and palpation, was used for $\mathrm{T}$ staging and proper discussion at the multidisciplinary tumor (MDT) board.

The superficial width of the lesion was preoperatively evaluated in the outpatient clinic by rigid endoscopy under white light (WL) and narrow-band imaging (NBI, Olympus Medical System Corporation, Tokyo, Japan). The deep extension of the tumor was assessed by imaging (IOUS and/ or MR) performed by dedicated radiologists. A supplementary detailed study of the neck was routinely performed with the US, possibly in association with fine-needle aspiration cytology in case of suspected lymph node metastases.

Intraoperative rigid endoscopy with $0^{\circ}$ telescopes under WL and NBI was repeated with the patient under general anesthesia to establish the appropriate surgical incision for radical excision [10]. All patients underwent complete surgical resection of the primary tumor with or without neck dissection according to NCCN guidelines [11]. For final data analysis, all tumors were homogeneously reclassified according to the 8th Edition of the AJCC UICC TNM Staging System [12]. Patients with biological risk factors such as perineural invasion, angioembolization, multiple lymph nodes metastases, pT4a category, poor differentiation, and/or extracapsular spread underwent adequate adjuvant treatment after MDT board discussion.

The entire cohort of patients received follow-up clinical examination every 2 months during the first 2 years, every 3 months during the third year, every 6 months in the fourth and fifth years, and then annually [11]. Radiological followup was performed twice in the first year and then annually for at least three years, even in the absence of suspicious clinical findings.

\section{MR}

MR was performed with a 1.5-T scanner (Aera, Siemens, Erlangen, Germany) and a 3.0-T scanner (Prisma, Siemens, Erlangen, Germany) with the manufacturer's phased-array head and neck coils. MR protocol included: axial, sagittal and coronal high-resolution turbo-SE T2 weighted sequences, slice thickness $3 \mathrm{~mm}$, axial turbo-SE T1 weighted sequences, coronal turbo-STIR, and axial echo-planar diffusion-weighted imaging (DWI) sequence with $b$ values of 50 and $800 \mathrm{~s} / \mathrm{mm}^{-2}$. Axial BLADE T2 weighted images with fat saturation were achieved in case of motion-related artifacts and a 3D fat saturated VIBE sequence with an isotropic 


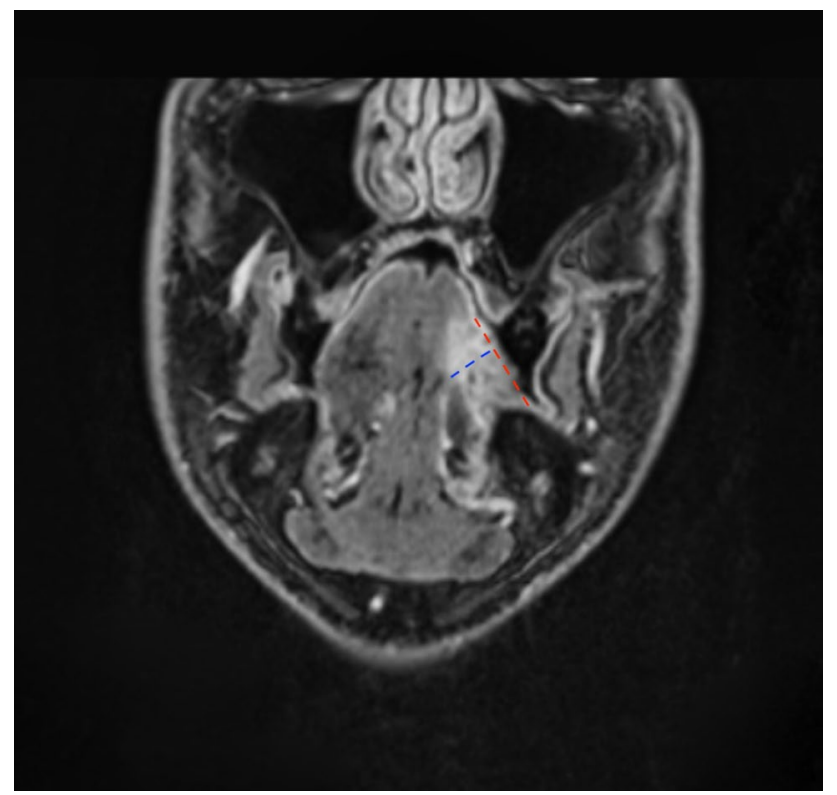

Fig. 1 Coronal-reformatted 3D T1-GRE acquisition showing an ulcerated squamous cell carcinoma of the oral tongue. $\mathrm{cDOI}_{\mathrm{MR}}$ was measured (blue dashed line) perpendicularly to the mucosal plane (red dashed line) pixel of $0.7 \mathrm{~mm}$ after paramagnetic contrast agent injection. Apparent diffusion coefficient (ADC) maps were automatically produced for each exam using a mono-exponential model. Two dedicated head and neck radiologists measured DOI independently, choosing the best sequence and plane in which tumor infiltration was depicted (Fig. 1). DOI was measured by drawing a plumb line to that connecting two edges of normal perilesional mucosa up to the most distant front of infiltration of the tumor deep in the tissue. If there was any mismatch between the measures reported by the two radiologists, the highest DOI value was considered and recorded as $\mathrm{cDOI}_{\mathrm{MR}}$.

\section{IOUS}

IOUS was performed by a dedicated head and neck radiologist using a hockey-stick high frequency (15-7 MHz) probe (Philips Healthcare, Philips North America Corporation, Andover, MA, USA) (Fig. 2a, b). A latex-free cover was filled with gel and placed on the probe. The lesion was fully explored to individuate the point of deepest infiltration. Next, to accurately measure its DOI, the probe was orientated along a plane perpendicular to the mucosal surface
Fig. 2 a DOI estimation (blue dashed line) in an exophytic SCC of the lateral border of the oral tongue. $\mathbf{b}$ Estimation of $\mathrm{cDOI}_{\text {IOUs }}$ in tongue carcinoma (blue dashed line). The dashed grey line represents the mucosal plane. The lesion was ulcerated: in such cases, a small amount of gel may be helpful to prevent air artifacts
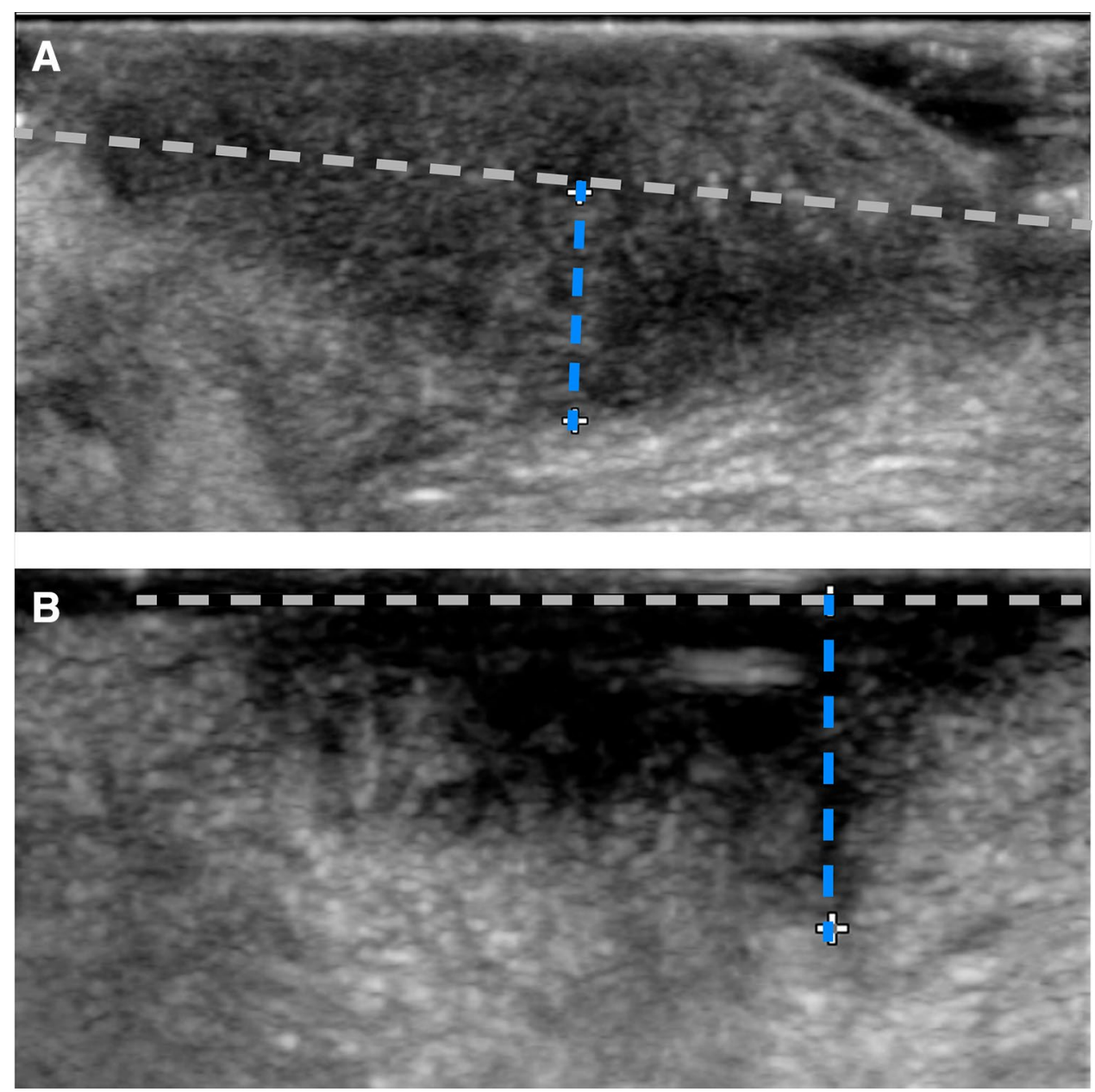
using light pressure in order not to alter tumor relationships with the mucosal plane or surrounding tissues. Several US images of the same lesion were recorded to obtain multiple

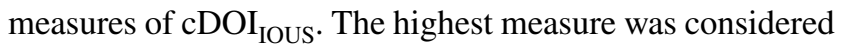
for comparison with the corresponding $\mathrm{cDOI}_{\mathrm{MR}}$.

\section{Statistical analysis}

Qualitative variables were described as absolute and relative frequencies; standard descriptive statistics were used for continuous variables, expressing means, medians, ranges and standard deviations. Shapiro-Wilk test was applied, testing normality distribution of continuous variables. Correlation analysis was performed using Spearman's rank correlation estimating confidence intervals by bootstrap with 1000 resamples, and paired comparison between methods of DOI measurement were tested by Wilcoxon Signed Rank test, as appropriate. The agreement in allocating tumors in different $\mathrm{T}$ categories within radiological techniques and compared to the pathological result was investigated by weighted Cohen's kappa. Cut-off optimization for $\mathrm{cDOI}_{\mathrm{MR}}$ and $\mathrm{cDOI}_{\mathrm{IOUS}}$ for the prediction of a target $\mathrm{pDOI} \geq 4 \mathrm{~mm}$ was performed by Receiver operating characteristic (ROC) curves analysis and maximization of Youden's index. In all analyses, two-tail tests with a significance level of 5\% were applied. R (version 3.6.3, R Foundation for Statistical Computing, Vienna, Austria) with the packages was used for statistical analysis and graphs drawing.

\section{Results}

Forty-nine patients (22 females, 27 males; mean age, 65.6 years; range, 22-95) met inclusion criteria. Nine (18.4\%) patients were staged by MR alone, 10 (20.4\%) by IOUS alone, and 30 (61.2\%) with both MR and IOUS (Table 1). Primary subsite was buccal mucosa in 6 patients, the floor of mouth in 4, and oral tongue in 39 (Table 1). The mean time elapsed between radiological imaging and surgery was 23.3 days (range, 1-67) for MR and 23.1 days (range, 1-118) for IOUS (Table 1). Further clinical and pathological $\mathrm{T}$ and $\mathrm{N}$ categories are reported in Table 1.

In the entire cohort, the mean value of DOI according to the different examinations was: $\mathrm{cDOI}_{\mathrm{MR}}, 7.2 \mathrm{~mm}(95 \%$ confidence interval [95\% CI] 5.3-9.2 mm); cDOI $_{\text {IOUS }}, 7.0 \mathrm{~mm}$ (95\% CI 5.4-8.6 mm); pDOI, $7.3 \mathrm{~mm}$ (95\% CI 5.2-9.4 mm) (Table 2). Spearman's rank correlation coefficient between MR and HIST was $R=0.83$ (95\% CI 0.64-0.94, $p<0.0001$ ), between IOUS and HIST it was $R=0.76$ (95\% CI 0.59-0.87, $p<0.0001)$ and in-between MR and IOUS $R=0.87(95 \%$ CI $0.74-0.94, p<0.0001)$ indicating good correlations, as reported in Fig. 3a-c and Table 3.
Table 1 Summary statistics of demographic and clinical variables

\begin{tabular}{|c|c|}
\hline & $\begin{array}{l}\text { Overall } \\
(N=49)\end{array}$ \\
\hline \multicolumn{2}{|l|}{ Age } \\
\hline Mean (SD) & $65.6(15.8)$ \\
\hline Median [Min, Max] & $65.0[22.0,95.0]$ \\
\hline \multicolumn{2}{|l|}{ Sex } \\
\hline $\mathrm{F}$ & $22(44.9 \%)$ \\
\hline M & $27(55.1 \%)$ \\
\hline \multicolumn{2}{|l|}{ Site } \\
\hline Buccal mocosa & $6(12.2 \%)$ \\
\hline Tongue & $39(79.6 \%)$ \\
\hline Floor of the mouth & $4(8.2 \%)$ \\
\hline \multicolumn{2}{|l|}{ cT category } \\
\hline $\mathrm{T} 1$ & $16(32.7 \%)$ \\
\hline $\mathrm{T} 2$ & $20(40.8 \%)$ \\
\hline $\mathrm{T} 3$ & $13(26.5 \%)$ \\
\hline \multicolumn{2}{|l|}{$\mathrm{cN}$ category } \\
\hline No & $39(79.6 \%)$ \\
\hline N1 & $2(4.1 \%)$ \\
\hline $\mathrm{N} 2 \mathrm{~b}$ & $5(10.2 \%)$ \\
\hline $\mathrm{N} 2 \mathrm{c}$ & $1(2.0 \%)$ \\
\hline $\mathrm{N} 3 \mathrm{~b}$ & $2(4.1 \%)$ \\
\hline \multicolumn{2}{|l|}{ pT category } \\
\hline $\mathrm{T} 1$ & $15(30.6 \%)$ \\
\hline $\mathrm{T} 2$ & $21(42.9 \%)$ \\
\hline $\mathrm{T} 3$ & $13(26.5 \%)$ \\
\hline \multicolumn{2}{|l|}{ pN category } \\
\hline N0 & $18(36.7 \%)$ \\
\hline N1 & $5(10.2 \%)$ \\
\hline $\mathrm{N} 2 \mathrm{~b}$ & $2(4.1 \%)$ \\
\hline $\mathrm{N} 2 \mathrm{c}$ & $2(4.1 \%)$ \\
\hline $\mathrm{N} 3 \mathrm{~b}$ & $4(8.2 \%)$ \\
\hline $\mathrm{Nx}$ & $18(36.7 \%)$ \\
\hline \multicolumn{2}{|c|}{$\Delta$ (Date surgery-Date $\mathrm{MR})$} \\
\hline Mean (SD) & $23.3(16.2)$ \\
\hline Median [Min, Max] & $20.0[1.00,67.0]$ \\
\hline Missing & $10(20.4 \%)$ \\
\hline \multicolumn{2}{|c|}{$\Delta$ (Date surgery-Date IOUS $)$} \\
\hline Mean (SD) & $23.1(21.8)$ \\
\hline Median [Min, Max] & $22.0[1.00,118]$ \\
\hline Missing & $9(18.4 \%)$ \\
\hline
\end{tabular}

In the entire cohort of patients matched measurements by MR, IOUS and histopathology were not significantly different, testing the null hypothesis of $\mathrm{cDOI}_{\mathrm{MR}}-\mathrm{pDOI}$, $\mathrm{cDOI}_{\text {IOUs }}-\mathrm{pDOI}$ and $\mathrm{cDOI}_{\mathrm{MR}^{-}} \mathrm{cDOI}_{\mathrm{IOUS}}$ to be 0 , by the Wilcoxon test ( $p=0.11, p=0.40$ and $p=0.88$, respectively, Fig. 1d, e and Table 2).

MR properly staged $64 \%$ of OCSCC lesions, while IOUS reached a perfect correspondence between $\mathrm{cT}$ and $\mathrm{pT}$ 
Table 2 Summary statistics of DOI measurements and differences among measurement techniques

\begin{tabular}{|c|c|}
\hline & $\begin{array}{l}\text { Overall } \\
(N=49)\end{array}$ \\
\hline \multicolumn{2}{|l|}{$\mathrm{cDOI}_{\mathrm{MR}}(\mathrm{mm})$} \\
\hline Mean (SD) & $7.22(6.02)$ \\
\hline Median [Min, Max] & $6.03[0,30.0]$ \\
\hline Missing & $10(20.4 \%)$ \\
\hline \multicolumn{2}{|l|}{$\mathrm{cDOI}_{\text {IOUS }}(\mathrm{mm})$} \\
\hline Mean (SD) & $7.00(5.08)$ \\
\hline Median [Min, Max] & $5.00[0,20.0]$ \\
\hline Missing & $9(18.4 \%)$ \\
\hline \multicolumn{2}{|l|}{ pDOI (mm) } \\
\hline Mean (SD) & $7.27(7.33)$ \\
\hline Median [Min, Max] & $6.00[0,40.0]$ \\
\hline \multicolumn{2}{|l|}{$\Delta\left(\mathrm{cDOI}_{\mathrm{MR}^{-}} \mathrm{pDOI}\right)$} \\
\hline Mean (SD) & $0.146(3.55)$ \\
\hline Median [Min, Max] & $1.04[-13.0,3.70]$ \\
\hline Missing & $10(20.4 \%)$ \\
\hline \multicolumn{2}{|l|}{$\Delta\left(\mathrm{cDOI}_{\mathrm{IOUS}}-\mathrm{pDOI}\right)$} \\
\hline Mean (SD) & $0.328(4.07)$ \\
\hline Median [Min, Max] & $0.500[-13.0,10.0]$ \\
\hline Missing & $9(18.4 \%)$ \\
\hline \multicolumn{2}{|l|}{$\Delta\left(\mathrm{cDOI}_{\mathrm{MR}^{-}}-\mathrm{CDOI}_{\mathrm{IOUS}}\right)$} \\
\hline Mean (SD) & $-0.0947(2.58)$ \\
\hline Median [Min, Max] & $0[-8.96,3.00]$ \\
\hline Missing & $19(38.8 \%)$ \\
\hline
\end{tabular}

categories in $50 \%$ of patients. Weighted Cohen's kappa test was applied to assess the agreement between MR or IOUS in allocating tumors to the corresponding final pT category, being the $\mathrm{k}$ coefficients 0.53 (95\% CI $0.32-0.74, p<0.0001$ ) and 0.35 (95\% CI $0.14-0.58, p=0.0015)$, respectively and in-between MR and IOUS 0.71 (95\% CI 0.53-0.90, $p<0.0001$ ), thus resulting in a good agreement between the two imaging techniques (Table 4 and Table S1).

Testing the detection of patients amenable to an elective neck dissection (pDOI $\geq 4 \mathrm{~mm}$ ), both MR and IOUS obtained good results with a sensitivity of $100 \%$ and $100 \%$, respectively; specificity of $73 \%$ and $47 \%$, positive predictive value of $86 \%$ and $72 \%$, negative predictive value of $100 \%$ and $100 \%$ and accuracy of $90 \%$ and $78 \%$, as reported with full details in Table 5 and Table S2. Furthermore, searching for the best cut-off of cDOI for a predicted pDOI $\geq 4 \mathrm{~mm}$, for both radiological technique it was $\geq 5 \mathrm{~mm}$, by Youden's index maximization (Fig. 4). The diagnostic test was satisfactory both for MR and IOUS with an Area under the curve (AUC) of 0.92 and 0.82 , respectively; a sensitivity of $92 \%$ and $87 \%$ and a specificity of $93 \%$ and $76 \%$, respectively (Fig. 4).

\section{Discussion}

We conducted this prospective study with the specific aim of investigating the performance of the emerging technique of intraoral ultrasonography (IOUS), compared to the gold standard represented by the magnetic resonance (MR) for the DOI evaluation in OCSCC. A similar investigation has been recently conducted by Noorlag and colleagues [13]; though, in their paper radiological TT values (instead of DOI values) from T1-T2 tongue cancers were compared with histopathological DOI.

The data herein show that both cDOI measured by IOUS and MR highly correlate with the pathological result (pDOI), even though MR obtained the best performance with a $R$ of 0.83 compared to 0.76 of IOUS as shown in Fig. 3. Similar results were obtained comparing the agreement of a correct $\mathrm{cT}$ category allocation, compared to the $\mathrm{pT}$ one, with a $k=0.53$ for MR and $k=0.36$ for IOUS. These differences could be due to the limits of IOUS to be a new and closely operator-dependent tool. Due to this fact, long-lasting and specific experience in head and neck US is needed to achieve optimal measurement of cDOI in OCSCC. On the contrary IOUS benefits of lower costs and easy application. Investigating the paired differences between the MR or IOUS results and the final pathological measure, matched measurements were not significantly different, meaning the absence of systematic over- or under-estimation of the pDOI for both radiological techniques (Fig. 1).

Nowadays, DOI should be considered as the most trustworthy criterion to be correlated with the threat of regional metastasization and prognostic outcomes in OCSCC. From the therapeutic point of view, cT1 and selected cT2 with cDOI $<10 \mathrm{~mm}$ [3], without major infiltration of the extrinsic musculature of the tongue, can be safely removed transorally. By contrast, for more advanced $\mathrm{T}$ categories, in the last two decades there has been progressive abandonment of circumferential and/or cuneiform resections in favor of longitudinal compartmental resection to ensure the best locoregional control and functional outcomes [14-17]. Moreover, cDOI, especially for cT1-cT2N0 lesions, represents an essential prognosticator in deciding whether to perform a simultaneous elective neck dissection or to delay it after the definitive histopathological report [18, 19]. It is well established that the probability of having occult nodal metastases in regional lymph nodes is mainly related to DOI. MohitTabatabai and colleagues [20] and Spiro and colleagues [21] first applied Breslow's hypothesis [22] with reference to the correlation between lymph node involvement and DOI in OCSCC. Nonetheless, to date, controversies still remain about the proper DOI cut-off for a clinically relevant risk of occult nodal metastases. Data from the literature have suggested that a cut-off could be set at $4 \mathrm{~mm}$ : consequently, 

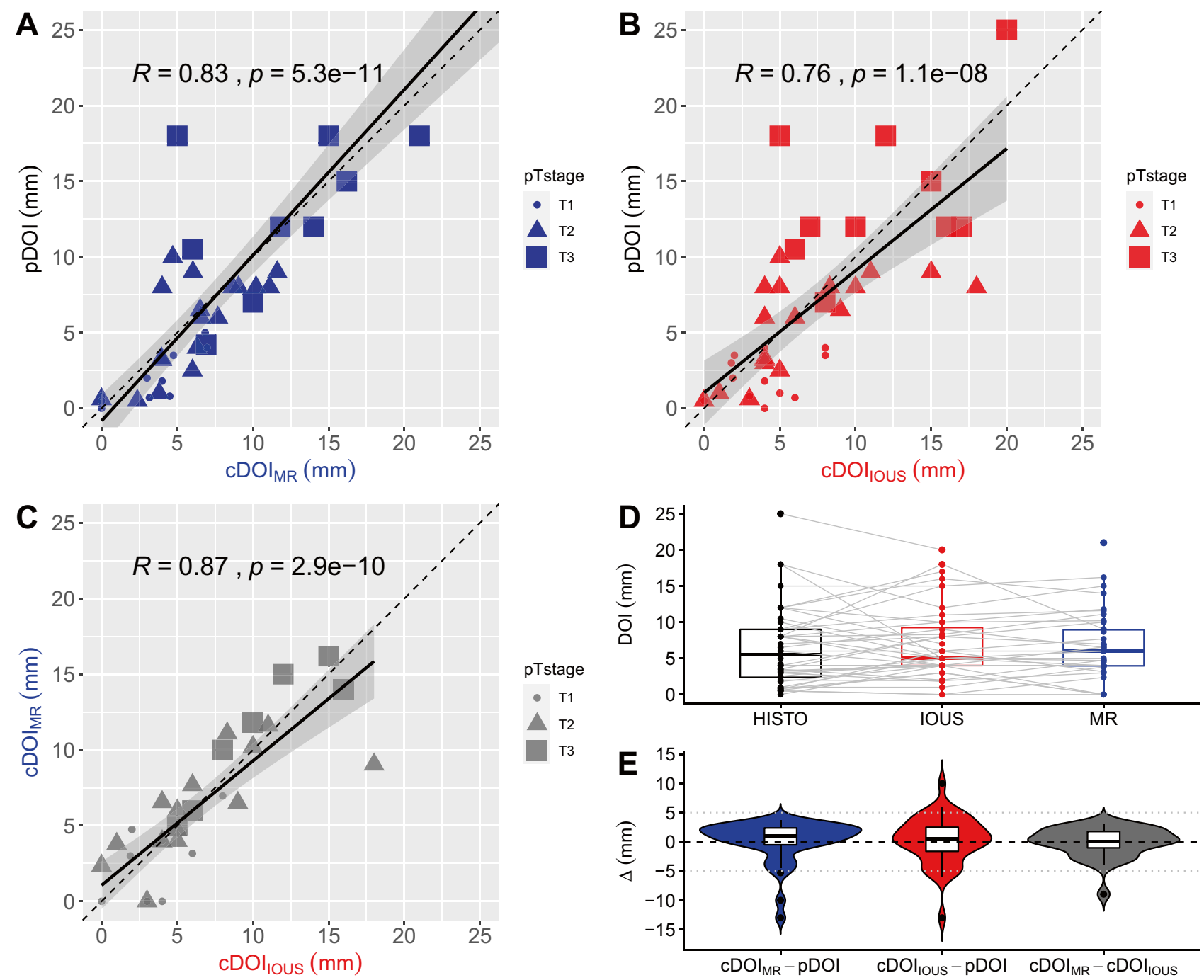

Fig. 3 Scatter plots showing the comparisons of pDOI with $\mathrm{cDOI}_{\mathrm{MR}}$ (a) or $\mathrm{cDOI}_{\mathrm{IOUS}}(\mathbf{b})$ and between $\mathrm{CDOI}_{\mathrm{MR}}$ and $\mathrm{cDOI}_{\mathrm{IOUS}}$ (c); plotted black linear regression line with $95 \%$ CI bands in shadow gray; pT

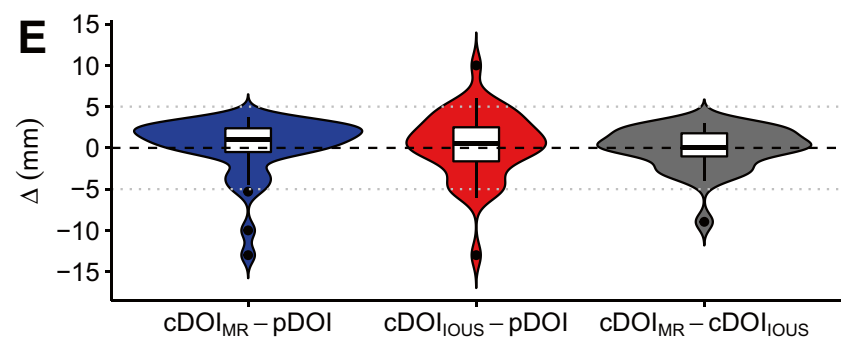

categories reported. Box plots showing paired DOI measures (d) and violin plots reporting differences distributions comparing the DOI assessed by different techniques

Table 3 Spearman correlation analysis of DOI measure among different techniques

\begin{tabular}{llll}
\hline & $\mathrm{pDOI}$ & $\mathrm{DOI}_{\mathrm{IOUS}}$ & $\mathrm{DOI}_{\mathrm{MR}}$ \\
\hline $\mathrm{DOI}_{\mathrm{MR}}$ & $R=0.83(0.64-0.94)$ & $R=0.87$ & 1 \\
& $p<0.0001$ & $(0.74-0.94)$ & \\
& & $p<0.0001$ & \\
DOI $_{\mathrm{IOUS}}$ & $R=0.76(0.59-0.87)$ & 1 & \\
& $p<0.0001$ & & \\
pDOI & 1 & & \\
\hline
\end{tabular}

cN0 patients with DOI $<4 \mathrm{~mm}$ could be safely spared from elective neck dissection [5-7]. Even if histopathological measurement of DOI remains the standard in the choice for prophylactic neck dissection in early OCSCC, its accurate

preoperative measurement allows performing, in selected cases, an elective neck dissection together with tumor resection, thus reducing the number of inappropriate overtreatments or two-step therapeutic approaches (i.e. tumor resection first and delayed neck dissection later). In our series both the evidence of a $\mathrm{cDOI}_{\mathrm{MR}}$ or $\mathrm{cDOI}_{\mathrm{IOUS}} \geq 4 \mathrm{~mm}$ obtained good results from the diagnostic test for the prediction of a pDOI $\geq 4 \mathrm{~mm}$ with an overall accuracy of $90 \%$ and $78 \%$, respectively, and a transversal Sensitivity and Negative predictive value of $100 \%$ for both techniques, meaning the correct identification of all patients truly amenable to an elective neck dissection and the almost perfect guarantee that a $\mathrm{cDOI}<4 \mathrm{~mm}$ corresponds to a pDOI $<4 \mathrm{~mm}$ too. Morover, investigating the best cut-off of $\mathrm{cDOI}_{\mathrm{MR}}$ or $\mathrm{cDOI}_{\mathrm{IOUS}}$ for the 
Table 4 Weighted Cohen's kappa test results between $\mathrm{T}$ categories applying different techniques of DOI measurement

\begin{tabular}{llll}
\hline & $\mathrm{pT}$ category & $\mathrm{cT}_{\text {IOUs }}$ category & $\mathrm{cT}_{\mathrm{MR}}$ category \\
\hline $\mathrm{cT}_{\mathrm{MR}}$ category & $k=0.53(0.32-0.74)$ & $k=0.71(0.53-0.90)$, & 1 \\
& $p<0.0001$ & $p<0.0001$ & \\
$\mathrm{cT}_{\text {IOUS }}$ category & $k=0.36(0.14-0.58)$ & 1 & \\
pT category & $p=0.0015$ & & \\
\hline
\end{tabular}

Table 5 Diagnostic test results of MR of IOUS assessment of DOI for the prediction of a $\mathrm{pDOI} \geq 4 \mathrm{~mm}$

\begin{tabular}{lcc}
\hline & MR & IOUS \\
& $\%\left(\mathrm{CI}_{95 \%}\right)$ & $\left(\mathrm{CI}_{95 \%}\right)$ \\
\hline Sensitivity & $100(86-100)$ & $100(85-100)$ \\
Specificity & $73(45-92)$ & $47(23-72)$ \\
Positive predictive value & $86(67-96)$ & $72(53-86)$ \\
Negative predictive value & $100(72-100)$ & $100(63-100)$ \\
Accuracy & $90(76-97)$ & $78(62-89)$ \\
\hline
\end{tabular}

correct prediction of a pDOI $\geq 4 \mathrm{~mm}$ this was $5 \mathrm{~mm}$ for both techniques, as shown in Fig. 4.

Furthermore, the role of pDOI as an independent indication for postoperative RT in early OCSCC still remains controversial [23]. In a recent multicenter study aimed at demonstrating if an increase in DOI parallels significant prognostic deterioration in patients with early OCSCC with no other adverse pathological characteristics, the authors reported that DOI was profoundly connected with other risk factors including involved or close surgical margins, dimensions of the primary tumor, pathological nodal staging, and extra-nodal extension [24]. Moreover, in a recent analysis of 1200 patients affected by OCSCC, the authors underlined the importance of DOI $>10 \mathrm{~mm}$ as an adjunctive indicator to deliver postoperative RT, even if it is not explicitly taken into account by NCCN guidelines [25].

All these findings clearly suggest the importance of accurate preoperative prediction of DOI in early-intermediate OCSCC. So far, MR has been shown to be the most accurate instrument for loco-regional staging of OCSCC, with a sensitivity of $94 \%$ according to the literature [26]. To date, a clear consensus in the literature concerning the best MR sequence with which to measure DOI is still lacking. Preda et al. [27] reported that contrast administration enhances tumor differently, depending on its size and vascularization, while in T2-weighted images lesions are predictably hyperintense. Some authors reported the use of different sequences to obtain the best measurement of the tumor: axial gadolinium-enhanced $\mathrm{T} 1$ sequence specifically for DOI, axial and coronal T2 and post-contrast T1 for the entire size of the lesion are proposed by Goel et al. [28]. On the other hand, Murakami et al. reported in their work that the selection of the optimal measurement protocol should be made on a case-by-case basis [29]. A significant issue that may negatively influence the accuracy of MR measurements is related to image quality due to the presence of possible motional artifacts. To resolve this issue, some authors [30] proposed the use of the BLADE sequence to reduce artifacts due to motion, pulsation [31], and dental implants [32].

Better performance of 3.0-T over 1.5-T MR is still a matter of debate in the literature. According to Moreno et al. [33] and Lu et al. [34], an expected signal-to-noise ratio increase proportional to the magnetic field strength is the most interesting characteristic of 3.0-T MR, but other features such as increased T1 relaxation time, decreased T2 relaxation time, increased magnetic susceptibility contrast, and increased spectral resolution for MR spectroscopy may also furnish important benefits. In contrast, Neumann et al. [35] noted some critical issues of 3.0-T MR due to the higher magnetic susceptibility that, according to the authors, led to possible spatial distortion. Singh et al. [36] reported the good agreement $(K=0.79)$ for $\mathrm{T}$ staging between MR and histopathology, with a change in the final pT category for only $14 \%$ of patients.

Baek et al. [37] reported on the utility of IOUS in prognosticating pathologic OCSCC TT. Furthermore, according to the authors, even if both CT and MR have some limitations in the evaluation of tongue cancers, MR gives more detailed information about soft tissue involvement compared to CT. According to Yesuratman et al. [18], preoperative IOUS shows a high correlation index with histopathology $(r=0.80)$, while MR demonstrates only a moderate one $(r=0.69)$. One of the major confounding factors in diminishing the trustworthiness of preoperative radiological evaluation is a recent biopsy. It is arduous for IOUS and MR to distinguish post-biopsy edema from squamous dysplasia and/or invasive SCC. In general, inflammation surrounding the tumor could blur the boundaries that are observed on MRI T2-weighted sequences, leading to a possible overestimation of DOI [29]. On the contrary, IOUS can provide some advantages due to the possibility of changing the orientation of the probe along different planes, even through the skin of the face if needed. Moreover, as practical tools to assess thin muscles and buccal fat layers, it can be helpful to ask the patient to protrude and move the tongue or swollen cheek. Nonetheless, to date, some disagreements persist with 


\section{A Sensitivity and specificity plot}

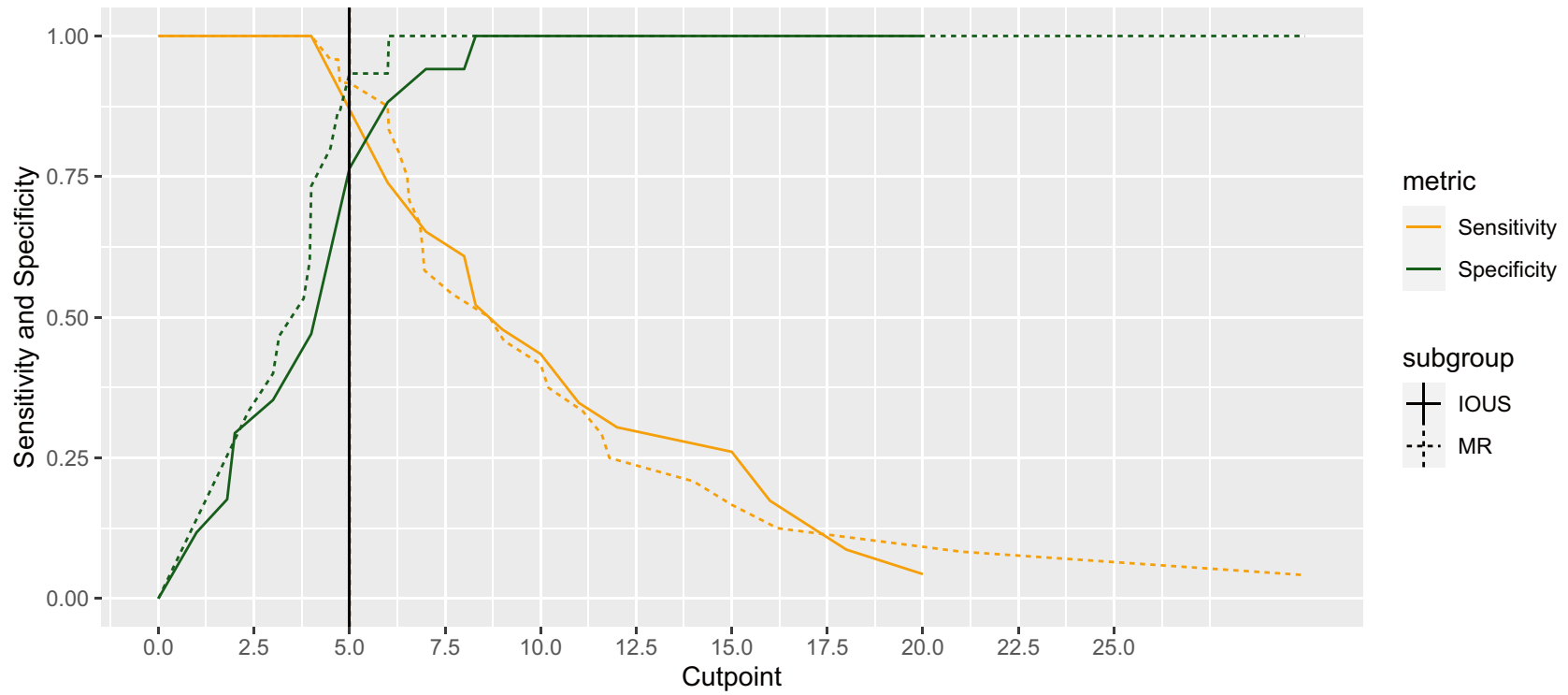

B ROC curve

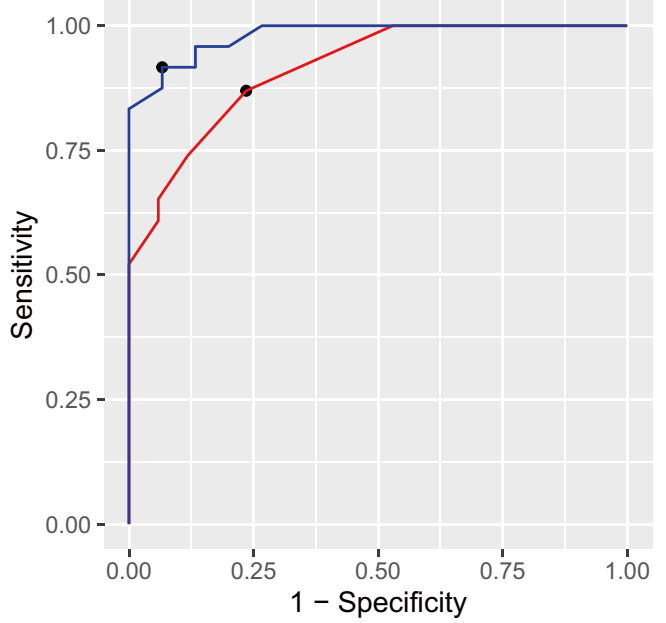

Fig. 4 a Plot showing the sensitivity and specificity changes, related to the correct detection of a pDOI $\geq 4 \mathrm{~mm}$ along with the variation of the cut-off for $\mathrm{cDOI}_{\mathrm{MR}}$ or $\mathrm{cDOI}$ IOUs. $\mathbf{b}$ Receiver operating characteristic (ROC) curve of $\mathrm{cDOI}_{\mathrm{MR}}$ of $\mathrm{cDOI}_{\mathrm{IOUS}}$ for $\mathrm{pDOI} \geq 4 \mathrm{~mm}$

regards to the best IOUS probe, the ideal probe frequency, and proper usage. In this regard, we chose a T-shape linear probe for the present study, following the suggestions of Iida and coworkers [38]. According to those authors, the best method to preoperatively measure DOI is the aforementioned probe inserted into a rubber sheath and filled with water. In this way, it is possibly best to outline the real 3D extent of the tumor, mainly because the same evaluation

\section{Independent variable (cDOI)}

optimal cutpoint and distribution by pDOI $>=/<4 \mathrm{~mm}$
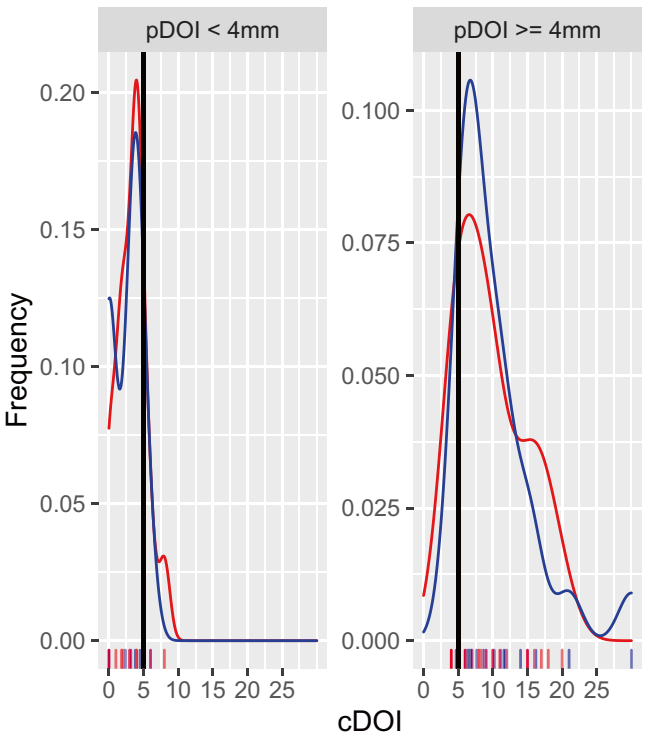

Subgroup

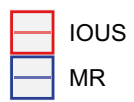

prediction showing the cut-off point of $5 \mathrm{~mm}$ for both techniques, by maximization of Youden's index. c Plots showing the distribution of $\mathrm{cDOI}_{\mathrm{MR}}$ and $\mathrm{cDOI}_{\mathrm{IOUS}}$ in $\mathrm{pDOI} \geq$ or $<4 \mathrm{~mm}$ groups, black vertical line at $5 \mathrm{~mm}$ of cDOI

may also be reproduced in the operating theater, just before the intervention.

The impossibility of attaining all oral subsites and the obstacle represented by adjacent bony structures appear to be the most considerable limitations of IOUS evaluation. As a consequence, tumors located in the posterior third of the mobile tongue are not easily accessible to perpendicular evaluation. Other IOUS technical issues are related to 
the fact that the probe should be kept in tight contact with the mucosal surface to be assessed to maximize the interface between them, trying not to exert an excessive pressure that could possibly distort or modify the aspect of the tumor with a consequent risk of underestimation of DOI. Moreover, IOUS is a live examination that is strictly dependent on the operator: as a consequence, variable levels of experience may give different information. As a somewhat new diagnostic tool, IOUS needs a proper learning curve to understand the adequate pressure to apply to tissues, the way to move the probe, and how to reach the more posterior subsites of the oral cavity. Finally, the same exam cannot be subsequently reassessed by a second operator nor visually aid surgeons before or during surgery.

Herein, both MR and IOUS had good correlation with histopathological findings and between them. For these reasons, we firmly believe that IOUS might be safely used, even alone, for preoperative staging of early OCSCC situated in the anterior half of the oral cavity.

The main strength of our study is represented by the homogeneous evaluation of the entire cohort of patients by the same two experienced head and neck radiologists. Moreover, the majority of patients included were evaluated by both preoperative MR and IOUS, allowing direct and fruitful comparison of the two radiological tools on the same lesion. The main concern of our findings is that our cohort of patients is relatively restricted and that the mean time dividing radiological and histopathological examination was almost 20 days.

\section{Conclusion}

At present, MR is considered the first-choice radiological examination for preoperative diagnostic assessment of head and neck SCCs, except for the larynx and hypopharynx. With MR, the radiologist is able to analyze neoplastic extension, intratumoral vascularization, tumor borders, and intracranial and/or perineural spreads. Its main limitations are that it is expensive, requires substantial time to perform a proper exam, and is impossible to perform in non-cooperative or claustrophobic patients, or in those with metallic prostheses and pacemakers. By contrast, the advantages of IOUS are related to the fact that is a fast and high-resolution examination, less invasive, more cost-effective, and requires less compliance by the patient. On the other hand, it remains highly operator-dependent and cannot be considered as the best radiological examination for lesions in close proximity to bony structures or located in the posterior half of the oral cavity. Notwithstanding, from our data it is possible to infer a good performance of IOUS and a good agreement with the current gold standard examination (MR). From these results, physicians may be encouraged to use IOUS as a complementary evaluation, being a less expensive and faster tool in the preoperative diagnostic work-up of earlyintermediate anterior OCSCC.

Funding Open access funding provided by Università degli Studi di Genova within the CRUI-CARE Agreement.

\section{Compliance with ethical standards}

Conflict of interest The authors certify that they have no affiliation with or involvement in any organization or entity with any financial interest.

Ethical approval The research did not involve any animal models; the research involved human participants in accordance with the ethical standards of the institutional and/or national research committees and with the 1964 Helsinki Declaration and its later amendments or comparable ethical standards; and informed consent was obtained from all individual participants included in the study. Ethical review and approval were not required for this study in accordance with the national and institutional requirements (Ethics committee CER Liguria). However, all patients preoperatively signed a consent form for disclosure of privacy in managing personal data for scientific purposes.

Informed consent Informed consent was obtained from all participants included in the study.

Open Access This article is licensed under a Creative Commons Attribution 4.0 International License, which permits use, sharing, adaptation, distribution and reproduction in any medium or format, as long as you give appropriate credit to the original author(s) and the source, provide a link to the Creative Commons licence, and indicate if changes were made. The images or other third party material in this article are included in the article's Creative Commons licence, unless indicated otherwise in a credit line to the material. If material is not included in the article's Creative Commons licence and your intended use is not permitted by statutory regulation or exceeds the permitted use, you will need to obtain permission directly from the copyright holder. To view a copy of this licence, visit http://creativecommons.org/licenses/by/4.0/.

\section{References}

1. Fitzmaurice C, Dicker D, Pain A et al (2015) The global burden of cancer 2013. JAMA Oncol 1:505-527

2. Edge SB, Byrd DR, Compton CC et al (2010) AJCC cancer staging manual, 7th edn. Springer, New York Dordrecht Heidelberg London, Chicago

3. Piazza C, Montalto N, Paderno A et al (2014) Is it time to incorporate "depth of infiltration" in the T staging of oral tongue and floor of mouth cancer? Curr Opin Otolaryngol Head Neck Surg 22:81-89

4. Melchers LJ, Schuuring E, van Dijk BAC et al (2012) Tumour infiltration depth $\geqslant 4 \mathrm{~mm}$ is an indication for an elective neck dissection in pT1cN0 oral squamous cell carcinoma. Oral Oncol 48:337-342

5. Brockhoff HC, Kim RY, Braun TM et al (2017) Correlating the depth of invasion at specific anatomic locations with the risk for regional metastatic disease to lymph nodes in the neck for oral squamous cell carcinoma. Head Neck 39:974-979. https://doi. org/10.1002/hed.24724 
6. Feng Z, Cheng A, Alzahrani S et al (2020) Elective neck dissection in T1N0M0 oral squamous cell carcinoma: when is it necessary? J oral Maxillofac Surg. https://doi.org/10.1016/j. joms.2020.06.037

7. van Lanschot CGF, Klazen YP, de Ridder MAJ et al (2020) Depth of invasion in early stage oral cavity squamous cell carcinoma: The optimal cut-off value for elective neck dissection. Oral Oncol 111:104940. https://doi.org/10.1016/j.oraloncology.2020.104940

8. Robertson AG, Soutar DS, Paul J et al (1998) Early closure of a randomized trial: surgery and postoperative radiotherapy versus radiotherapy in the management of intra-oral tumours. Clin Oncol (R Coll Radiol) 10:155-160

9. Grégoire V, Lefebvre J-L, Licitra L et al (2010) Squamous cell carcinoma of the head and neck: EHNS-ESMO-ESTRO Clinical Practice Guidelines for diagnosis, treatment and follow-up. Ann Oncol 21:184-186

10. Farah CS, Dalley AJ, Nguyen P et al (2016) Improved surgical margin definition by narrow band imaging for resection of oral squamous cell carcinoma: a prospective gene expression profiling study. Head Neck 38:832-839. https://doi.org/10.1002/hed.23989

11. Pister D, Spencer S, Adelstein D (2019) NCCN guidelines version 3.2019. Head and Neck Cancer. https://www.nccn.org/profession als/physician_gls/pdf/head-and-neck.pdf

12. Brierley JD, Gospodarowicz MK, Wittekind C (2017) TNM Classification of Malignant Tumours, 8th edition, Eight Edit. John Wiley and Sons

13. Noorlag R, Klein Nulent TJW, Delwel VEJ et al (2020) Assessment of tumour depth in early tongue cancer: accuracy of MRI and intraoral ultrasound. Oral Oncol 110:104895. https://doi. org/10.1016/j.oraloncology.2020.104895

14. Sanders I, Mu L (2013) A three-dimensional atlas of human tongue muscles. Anat Rec. https://doi.org/10.1002/ar.22711

15. Calabrese L, Bruschini R, Giugliano G et al (2011) Compartmental tongue surgery: long term oncologic results in the treatment of tongue cancer. Oral Oncol 47:174-179

16. Piazza C, Grammatica A, Montalto N et al (2019) Compartmental surgery for oral tongue and floor of the mouth cancer: oncologic outcomes. Head Neck 41:110-115. https://doi.org/10.1002/ hed. 25480

17. Grammatica A, Piazza C, Montalto N et al (2020) Compartmental surgery for oral tongue cancer: objective and subjective functional evaluation. Laryngoscope. https://doi.org/10.1002/lary.28627

18. Yesuratnam A, Wiesenfeld D, Tsui A et al (2014) Preoperative evaluation of oral tongue squamous cell carcinoma with intraoral ultrasound and magnetic resonance imaging-Comparison with histopathological tumour thickness and accuracy in guiding patient management. Int J Oral Maxillofac Surg 43:787-794

19. Jung J, Cho NH, Kim J et al (2009) Significant invasion depth of early oral tongue cancer originated from the lateral border to predict regional metastases and prognosis. Int J Oral Maxillofac Surg 38:653-660

20. Mohit-Tabatabai MA, Sobel HJ, Rush BF, Mashberg A (1986) Relation of thickness of floor of mouth stage I and II cancers to regional metastasis. Am J Surg 152:351-353

21. Spiro RH, Huvos AG, Wong GY et al (1986) Predictive value of tumor thickness in squamous carcinoma confined to the tongue and floor of the mouth. Am J Surg 152:345-350

22. Breslow A (1975) Tumor thickness, level of invasion and node dissection in stage I cutaneous melanoma. Ann Surg 182:572-575

23. Ivaldi E, Di Mario D, Paderno A et al (2019) Postoperative radiotherapy (PORT) for early oral cavity cancer (pT1-2, N0-1): a review. Crit Rev Oncol Hematol 143:67-75. https://doi. org/10.1016/j.critrevonc.2019.08.003
24. Ebrahimi A, Gil Z, Amit M et al (2019) Depth of invasion alone as an indication for postoperative radiotherapy in small oral squamous cell carcinomas: An International Collaborative Study. Head Neck 41:1935-1942. https://doi.org/10.1002/hed.25633

25. Lin C-Y, Fan K-H, Lee L-Y et al (2019) Precision adjuvant therapy based on detailed pathological risk factors for resected oral cavity squamous cell carcinoma: long term outcome comparison of CGMH and NCCN guidelines. Int J Radiat Oncol. https://doi. org/10.1016/j.ijrobp.2019.08.058

26. Dhoot NM, Hazarika S, Choudhury B et al (2015) Evaluation of tongue cancer using high-resolution sonography. comparison with magnetic resonance imaging. J Ultrasound Med 34:1537-1546

27. Preda L, Chiesa F, Calabrese L et al (2006) Relationship between histologic thickness of tongue carcinoma and thickness estimated from preoperative MRI. Eur Radiol 16:2242-2248. https://doi. org/10.1007/s00330-006-0263-9

28. Goel V, Parihar PS, Parihar A et al (2016) Accuracy of MRI in prediction of tumour thickness and nodal stage in oral tongue and gingivobuccal cancer with clinical correlation and staging. J Clin Diagnostic Res 10:TC01-TC05

29. Murakami R, Shiraishi S, Yoshida R et al (2019) Reliability of MRI-derived depth of invasion of oral tongue cancer. Acad Radiol 26:e180-e186. https://doi.org/10.1016/j.acra.2018.08.021

30. Finkenzeller T, Wendl CM, Lenhart S et al (2015) BLADE sequences in transverse $\mathrm{T} 2$-weighted $\mathrm{MR}$ imaging of the cervical spinecut-off for artefacts? Rofo 187:102-108. https://doi. org/10.1055/s-0034-1385179

31. Lavdas E, Vlychou M, Zaloni E et al (2015) Elimination of motion and pulsation artifacts using BLADE sequences in shoulder MR imaging. Skeletal Radiol 44:1619-1626. https://doi.org/10.1007/ s00256-015-2232-3

32. Cassetta M, Pranno N, Stasolla A et al (2017) The effects of a common stainless steel orthodontic bracket on the diagnostic quality of cranial and cervical 3T- MR images: a prospective, case-control study. Dentomaxillofacial Radiol 46:1-13. https:// doi.org/10.1259/dmfr.20170051

33. Moreno KF, Cornelius RS, Lucas FV et al (2017) Using 3 Tesla magnetic resonance imaging in the pre-operative evaluation of tongue carcinoma. J Laryngol Otol 131:793-800

34. Lu H, Nagae-Poetscher LM, Golay X et al (2005) Routine clinical brain MRI sequences for use at 3.0 Tesla. J Magn Reson Imaging 22:13-22

35. Neumann JO, Giese H, Biller A et al (2015) Spatial distortion in MRI-guided stereotactic procedures: evaluation in 1.5-, 3- and 7-Tesla MRI Scanners. Stereotact Funct Neurosurg 93:380-386. https://doi.org/10.1159/000441233

36. Singh A, Thukral CL, Gupta K et al (2017) Role of MRI in evaluation of malignant lesions of tongue and oral cavity. Polish J Radiol 82:92-99

37. Baek CH, Son YI, Jeong HS et al (2008) Intraoral sonographyassisted resection of T1-2 tongue cancer for adequate deep resection. Otolaryngol Head Neck Surg 139:805-810. https://doi. org/10.1016/j.otohns.2008.09.017

38. Iida Y, Kamijo T, Kusafuka K et al (2018) Depth of invasion in superficial oral tongue carcinoma quantified using intraoral ultrasonography. Laryngoscope 128:2778-2782

Publisher's Note Springer Nature remains neutral with regard to jurisdictional claims in published maps and institutional affiliations. 